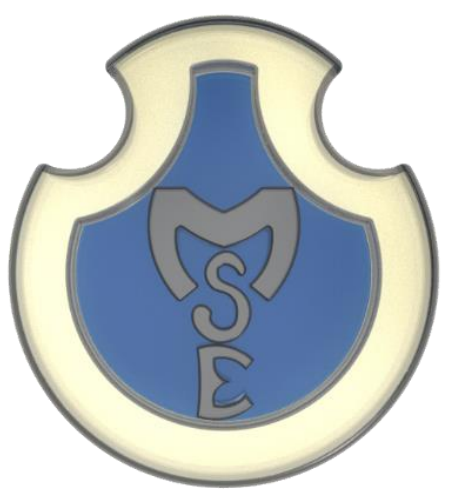

PREFACE

The $10^{\text {th }}$ International Conference on Manufacturing Science and Education - MSE 2021 was organized by the Industrial Engineering and Management Department and Industrial Machines and Equipment Department from Faculty of Engineering - Lucian Blaga University of Sibiu, Romania during 2 - 4 June, 2021.

The conference website is located at http://site.conferences.ulbsibiu.ro/mse/.

The goal of MSE 2021 Conference was to bring together specialists from universities, institutions and companies to provide an opportunity for the exchange of knowledge and ideas regarding trends in new industrial revolution, to promote modern and innovating manufacturing processes and systems as well as modern educational methods used in technical universities.

Some raw numbers are as follows: $\mathbf{2 1 6}$ papers were received and $\mathbf{1 5 1}$ of them have been accepted to be presented at the conference. All the submitted papers were subjected to a rigorous peer review process, each paper being reviewed by minimum two members of the Reviewing Committee. The accepted papers works have been presented ON-LINE at MSE 2021 Conference and people from 16 countries have been involved as authors and co-authors.

Finally, we would like to thank all people who have been supportive to MSE 2021 Conference: the expert refers whose enormous work for assessing the papers is gratefully appreciated, the invited keynote speakers and those who serve as chairmen for their invaluable contribution, the authors themselves for contributing research papers without whose expert input there would have been no conference, the sponsor organizations for their financial sponsorship, the members of the International and Organizing Committee that have been extremely helpful in successfully organizing of the conference and last but not least, we would like to express our gratitude to MATEC team for its support in online publishing of MSE 2021 Proceedings.

We consider that the $10^{\text {th }}$ International Conference on Manufacturing Science and Education - MSE 2021 has been a stimulating and memorable conference that has generated numerous fruitful discussions and layed the foundations of new future collaborations.

Chairman of MSE 2021 Conference

Prof. Eng. Nicolae Florin COFARU, PhD. 


\title{
HONORARY COMMITTEE
}

BANABIC Dorel, Romania BERCE Petru, Romania BRÎNDAŞU Paul Dan, Romania
DRĂGHICI George, Romania DUŞE Dan Maniu, Romania OPREAN Constantin, Romania

\section{ORGANIZING COMMITTEE}

\author{
CHAIRPERSON
}

Nicolae Florin COFARU

\section{MAIN ORGANIZERS}

Valentin GRECU

Dorin VLAD

Marinela INŢĂ
Radu Emanuil PETRUSE

Horia Andrei BRĂNESCU

\section{MEMBERS}

Alexandru BÂRSAN

Livia Dana BEJU

Marius BIBU

Cristina BIRIȘ

Ioan BONDREA

Radu-Eugen BREAZ

Anca CHICEA

Lucian-Ionel CIOCA

Florin $\mathrm{CIOFU}$

Ileana Ioana COFARU

Mihai CRENGANIS

Cristian DEAC

Dan DOBROTĂ

Dănuț-Dumitru DUMITRAȘCU

Carmen DUȘE

Fineas MORARIU

Claudia GÎRJOB

Claudiu Vasile KIFOR

Lucian LOBONT
Iosif Adrian MAROȘAN

Dan MIRICESCU

Gina-Maria MORARU

Mihaela OLEKSIK

Valentin OLEKSIK

Adrian PASCU

Valentin PETRESCU

Daniela POPA

Maria Cristina POPA

Liliana Georgeta POPESCU

Mihai POPP

Carmen-Maria PURCAR

Sever Gabriel RACZ

Liviu ROSCA

Mihaela ROTARU

Gabriela RUSU

Carmen SIMION

Melania TERA

Mihail TÎȚU 


\section{INTERNATIONAL SCIENTIFIC COMMITTEE}

\author{
AGRUSTI Gabriella, Italy \\ Ahrens Andreas, Germany \\ AKOS Cziszar, Germany \\ ALEGRE de la ROSA Olga María, Spain \\ ARSLAN Hasan, Turkey \\ AVASILCĂI Silvia, Romania \\ AVRAM Mihai, Romania \\ BADEA Adrian, Romania \\ BĂDESCU Mircea, Romania \\ BALAN Tudor, France \\ BÂLC Nicolae Octavian, Romania \\ BANCIU Felicia, Romania \\ BANICA Mihai, Romania \\ BARANOWSKA Wanda, Poland \\ BÂRSAN Ghiţă, Romania \\ BATOG Jacek, Poland \\ BEJINARIU Costică, Romania \\ BEJU Livia Dana, Romania \\ BELU Radian, USA \\ BENIAK Juraj, Slovakia \\ BERNEVIG Mihai-Adrian, Romania \\ BIBU Marius, Romania \\ BIRIS Cristina, Romania \\ BIRO Istvan, Hungary \\ BLAGA Florin, Romania \\ BLAGOJEVIĆ Mirko, Serbia \\ BLICHARSKI Jacek, Poland \\ BOLOGA Octavian, Romania \\ BONDREA Ioan, Romania \\ BOROIU Alexandru, Romania \\ BORZAN Marian, Romania \\ Brad Stelian, Romania \\ BRATU Danteş Nicolae, Romania \\ BREAZ Radu Eugen, Romania \\ BUNGĂU Constantin, Romania \\ CAMPBELL R Jan, United Kingdom \\ CAPPERUCCI Davide, Italy \\ CAREAN Alexandru, Romania \\ CERNUŞCĂ Dan, USA \\ CHIOU Richard, USA \\ CIOCA Lucian Ionel, Romania \\ CIOCA Marius, Romania \\ CIUPERCA Rodion, Moldova \\ COCÂRȚĂ Diana, Romania \\ COFARU Nicolae, Romania \\ CONSTANTIN George, Romania \\ COTET Costel Emil, Romania \\ CRETU Daniela, Romania \\ CROITORU Sorin, Romania \\ CUKOVIC Sasa, Serbia \\ CZERNIACHOWICZ Barbara, Poland \\ DARABONT Doru Costin, Romania \\ DAYNIER Rolando Delgado Sobrino, \\ Slovakia \\ DEAC Cristian, Romania \\ DENEŞ Călin, Romania \\ DETTORI Giuseppe Filippo, Italy \\ DEZSÖ Gergely, Hungary \\ DOBRESCU Tiberiu, Romania \\ DOBROTA Dan, Romania
}

DODUN Oana, Romania

DOICIN Cristian, Romania

DRĂGHICI Anca, Romania

DRĂGOI Mircea, Romania

DRAGOMIR MIHAI, Romania

DRÉGELYI-KISS Ágota, HUNGARY

DUDESCU CRISTIAN, Romania

DUMITRAȘ Cătălin Gabriel, Romania

DUMITRAŞCU Dănuţ, Romania

DUMITRU Ilie, Romania

DUŞE Carmen Sonia, Romania

FABBRICINO Massimiliano, Italy

FETECĂU Cătălin, Romania

FILIP Alexandru, Romania

FRANZ Charles, USA

Gabor Aron, France

GÁL József, Hungary

GĂMAN George Artur, Romania

GAVRUS Adinel, France

GIRJOB Claudia, Romania

GONET Andrzej, Poland

GORECKY Dominic, Germany

GÓRNY Adam, Poland

GRABARA Janusz, Poland

GRECU VALENTIN, Romania

GRUIONU Lucian, Romania

HADAR Anton, Romania

HEINISCH Martin, Germany

HOLUBEK Radovan, Slovakia

HORODINCĂ Mihăiţă, Romania

HUANG Regina, China

HUSI Géza, Hungary

HUSSAIN Hafezali Iqbal, Malaysia

ILIEŞ Remus, Singapore

ILIEVA Galina, Bulgaria

INȚĂ Marinela, Romania

IVAŞCU Larisa, Romania

KIFOR Claudiu, Romania

KOPCZYŃSKI Artur, POLAND

KOSTAL Peter, Slovakia

KOT Sebastian, South Africa

KRÁLIKOVÁ Ružena, Slovakia

Kuehn Martin, Germany

KUZMANOVIĆ Siniša, Serbia

KYRATSIS Panagiotis, Greece

ŁACIAK Mariusz, Poland

LANG Jürgen, Germany

LAREK Roland, Germany

LEGUTKO Stanislaw, Poland

LOBONT Lucian, Romania

LOBONTIU Mircea, Romania

LÓPEZ-ESCALANTE María Cruz, Spain

LUCA Razvan, Germany

LUNGU Florin, Romania

MAG Alina, Romania

MARA Daniel, Romania

MARICIC Sven, Croatia

MARJANOVIĆ Nenad, Serbia

MARSAVINA Liviu, Romania

MĂSĂLAR Liviu, Belgium 


\author{
MEIXNER Gerrit, Germany \\ MILOVANOVIĆ Dobrica, Serbia \\ MILTENOVIĆ Aleksandar, Serbia \\ MILTENOVIĆ Vojislav, Serbia \\ MIRICESCU Dan, Romania \\ MITROVIĆ Radivoje, Serbia \\ MOCAN Marian, Romania \\ MOHORA Cristina, Romania \\ MOLINOS-SENANTE Maria, Chile \\ MORARU Cristin Olimpiu, Romania \\ MORARU Roland Iosif, Romania \\ MOREY Merce, Spain \\ MORO Nicolae, Romania \\ MOSER Thomas, Austria \\ MUNTEAN Achim, Romania \\ NAGÎT Gheorghe, Romania \\ NAGY Stanisław, Poland \\ NEDELCU Dumitru, Romania \\ OANCEA Gheorghe, Romania \\ OLEKSIK Valentin, Romania \\ Papathanasiou Jason, Greece \\ PASCU Adrian, Romania \\ PASTRAMA Stefan, Romania \\ PETRESCU Valentin, Romania \\ PETRUSE Radu Emanuil, Romania \\ PÎRVU Bogdan-Constantin, Romania \\ PISLA Doina, Romania \\ POPESCU Daniela, Romania \\ PUŞCĂ Daniela, Canada \\ RACKOV Milan, Serbia \\ RACOVITZA Alexandru, Romania \\ RACZ Sever Gabriel, Romania \\ RADA Elena Cristina, Italy \\ RADOVANOVIĆ Miroslav, Serbia \\ RADU Sorin Mihai, Romania \\ RAGAZZI Marco, Italy \\ RATH Setik, Cambodia
}

ROSCA Adrian Sorin, Romania ROŞCA Liviu, Romania ROTHMUND Johannes, Germany RUZAROVSKY Roman, Slovakia RYBICKI Czesław, Poland SARFRAZ Muddassar, China SAROSI Jozsef, Hungary SAVU Tom, Romania SHOOSHTARI Nader H., USA SIEMEK Jakub, Poland SIMION Carmen Mihaela, Romania SLATINEANU Laurentiu, Romania SLOMKA Tadeusz, Poland SOM Ratana, Cambodia Staak Anthony, South Africa STAN Simona, USA ŞTEFĂNESCU Dan Paul, Romania ȘTEFĂNESCU Mariana - Florentina, Romania TABAKOVIC Slobodan, Serbia ȚÎTU Mihail Aurel, Romania TORRETTA Vincenzo, Italy TRIF Adrian, Romania TROTMAN Dave, United Kingdom UDROIU Razvan, Romania ULIASZ-MISIAK Barbara, Poland UNGUREANU Nicolae, Romania VELISEK Karol, Slovakia VENTURA Marina, Portugal WISNIOWSKI Rafał, Poland ZAHARIA Sebastian, Romania Zajko Marian, Slovakia ZELJKOVIĆ Milan, Serbia ZUHLKE Detlef, Germany 


\section{REVIEWING COMMITTEE}

AGRUSTI Gabriella

AHRENS Andreas

MARÍA ALEGRE DE LA ROSA Olga

ARSLAN Hasan

AVASILCĂI Silvia

AVRIGEAN Eugen

BANABIC Dorel

BANCIU Felicia Veronica

BĂDESCU Mircea

BĂNICĂ Mihai

BANU Mihaela

BÂRSAN Raluca

BATÓG Jacek

BEJINARIU Costică

BEJU Livia

BENIAK Juraj

BERNEVIG Mihai-Adrian

BIBU Marius

BIRIȘ Cristina

BLAGA Florin

BLAGOJEVIC Mirko

BLICHARSKI Jacek

BOGDAN Mihai

BOLOGA Octavian

BONDREA Ioan

BOROIU Alexandru

BOSTAN Ionel

BRĂNESCU Andrei Horia

BREAZ Radu-Eugen

BUNGĂU Constantin

BUTĂNESCU-VOLANIN Remus

Constantin

CERNUȘCĂ Dan

CHICEA Anca

CHIRIȚĂ Bogdan

CIOCA Lucian-Ionel

CIOCA Marius

CIOFU Florin

COCÂRȚĂ Diana Mariana

COFARU Ileana Ioana

COFARU Nicolae Florin

CONSTANTIN George

COTET Costel Emil

CRENGĂNIS Mihai

CREȚU Daniela

CSISZAR Akos

CUKOVIC Sasa

CZERNIACHOWICZ Barbara

DAN Nicoleta

DARABONT Doru Costin

DEAC Cristian

DENES Călin

DOBROTĂ Dan

DODUN Oana

DRĂGHICI Anca

DRĂGHICI George

DRĂGOI Mircea Viorel

DRAGOMIR Mihai
DRÉGELYI-KISS Ágota

DUDESCU Cristian

DUMITRAȘ Catalin Gabriel

DUMITRAȘCU Dănuț Dumitru

DUMITRAȘCU Manuel

DUMITRU Ilie

DUȘE Carmen Sonia

FETECĂU Catalin

FILIP Alexandru

FOIDAȘ Ion

GAL Marius

GAVRUS Adinel

GHEORGHE Viorel

GÎRJOB Claudia

GIUREA Ramona

GORECKY Dominic

GÓRNY Adam

GRECU Valentin

GRUIONU Lucian

HEINISCH Martin

HOLUBEK Radovan

HUSI Geza

IANOSI-ANDREEVA-

DIMITROVA Alexandru

INȚĂ Marinela

ISARIE Claudiu Laurențiu

IVAȘCU Larisa

KIFOR Claudiu

KOSTAL Peter

KYRATSIS Panagiotis

LEGUTKO Stanislaw

LOBONT Lucian

LOBONȚIU Mircea

LUNGU Florin

LUPU Diana-Andreea

MAG Alina Georgeta

MÂNDRU Dan

MARA Daniel

MARICIC Sven

MARJANOVIC Nenad

MAZĂRE Alin Gheorghiță

MEIXNER Gerrit

MILTENOVIC Aleksandar

MIRICESCU Dan

MOCAN Marian

MOHORA Cristina

MORARU Gina-Maria

MORARU Roland Iosif

MOREY Merce

NAGÎȚ Gheorghe

NICOLAESCU Sergiu Ștefan

NITUUESCU Mircea

OANCEA Gheorghe

OLEKSIK Mihaela

OLEKSIK Valentin

PAPATHANASIOU Jason

PASCU Adrian

PĂSCULESCU Vlad Mihai 
PASTRAMĂ Stefan Dan

PĂUNOIU Viorel

PETRESCU Valentin

PETRUSE Radu

PÎRVU Bogdan-Constantin

POPA Daniela

POPESCU Dorin

POPESCU Liliana Georgeta

POPESCU-STELEA Mihai

POPP Ilie

PRODEA Laurentiu

PURCAR Carmen

RACKOV Milan

RACOVITZA Alexandru

RACZ Sever-Gabriel

RĂULEA Andreea

ROȘCA Liviu

ROȘCA Adrian Sorin

ROTARU Mihaela

RUZAROVSKY Roman

SARFRAZ Muddassar

SEVERIN Traian

SIMA Elena

SIMION Carmen
SLĂTINEANU Laurențiu

SOM Ratana

STAN Simona

ŞTEFĂNESCU Dan Paul

ȘTEFĂNESCU Mariana-Florentina

SUCIU Cornel

TABAKOVIĆ Slobodan

TĂTAR Mihai Olimpiu

TERA Melania

ȚîTU Mihail Aurel

TRIF Adrian

UDROIU Răzvan

ULIASZ-MISIAK Barbara

UNGUREANU Nicolae

VASILESCU Dragoș Gabriel

VENTURA Marina

ZAHARIA Sebastian Marian

ZAJKO Marian

ZELJKOVIĆ Milan

ZERBES Mihai 


\section{INVITED KEYNOTE SPEAKERS}

\section{Dorel BANABIC}

Romanian Academy

Recent Developments in The Simulation and Modelling of Sheet Metal Forming Processes

\section{Ian CAMPBELL}

Loughborough University

How Design for Additive Manufacturing has Created New Product Design

Opportunities

\section{Anca DRĂGHICI}

Politehnica University of Timişoara, Romania

Multimedia Competencies for University Staff to Empower Manufacturing Science

Education

Nicolae BẤLC

Technical University of Cluj-Napoca, Romania

3D Printing Applications and Perspectives 


\title{
PARALLEL SESSIONS
}

SESSION 1 - Design, Manufacturing and Management of Machine Elements, Technological Equipment and Systems

SESSION 2 - Management, Modelling and Monitoring of Manufacturing Processes

SESSION 3 - Advanced Manufacturing Technologies

SESSION 4 - Computer-aided technologies (CAD/CAM/CAE - CAx), Virtual Engineering and Reverse Engineering

SESSION 5 - Quality Engineering and Management

SESSION 6 - Transport Engineering and Road Vehicles, Traffic Management

SESSION 7 - Management and Economic Engineering

SESSION 8 - Mechanical Engineering, Mechatronics and Robotics

SESSION 9 - Engineering in Oil and Gas Field, Environmental Engineering

SESSION 10 - Safety and Health at Work

SESSION 11 - Academic Education and Management

\section{WORKSHOPS}

\author{
WORKPLACE SOCIAL INCLUSION, OCCUPATIONAL SAFETY AND ENVIRONMENTAL \\ RISK ASSESSMENT \\ Chairpersons: Diana Cocârță, Maria Cruz Lopez Escalante, Massimiliano Fabbricino, Lucian-Ionel \\ Cioca
}

